\title{
Low-Profile Planar Eleven Antenna Over a Magnetic Plane
}

\author{
Abolfazl Haddadi ${ }^{1}$, Parastoo Taghikhani ${ }^{1}$, Jian Yang $^{2}$, Per-Simon Kildal ${ }^{2}$ \\ ${ }^{1}$ Department of Electrical Engineering, AmiKabir University of Technology, Tehran, Iran \\ ${ }^{2}$ Department of Signals and Systems, Chalmers University of Technology, Gothenburg, Sweden \\ Emails: haddadi_givi@aut.ac.ir, taghikhani@aut.ac.ir, jian.yang@chalmers.se,per-simon.kildal@chalmers.se
}

\begin{abstract}
The eleven antenna has been greatly used in many applications to achieve low reflection coefficient and stablepattern over ultra-wide-bands. However, arraying in the eleven configuration forces the antenna designer to tilt the antenna over an electric conducting ground plane, and this makes it mechanically complicated and increases the profile of the antenna. Seeking new capabilities, the theory of putting logperiodic dipoles in the eleven configuration over magnetic ground is developed. The eleven antenna is formed by two printed foldeddipoles, and we evaluate the performance for a single-pair of dipoles and five-pairs of dipoles. The results are extracted for the cases of PEC ground, PMC ground and without ground. The results are in a good agreement with theory. Also, some discussions are provided for the realization of the artificialmagnetic-ground.
\end{abstract}

Index Terms - eleven antenna, perfect magnetic conductor, lowprofile, ultra-wide-band.

\section{INTRODUCTION}

The eleven antenna as a log-periodic dipole antenna, is one of the solutions to demands on stable pattern and directivity over wider band-widths. In many applications like radioastronomy, remote-sensing, radar and space-communications, we need to have a feed antenna that shows more than decade bandwidths. For example, The Square Kilometer Array (SKA) radio telescopes need reflector antennas, covering $0.35-24 \mathrm{GHz}$ or more [1]. The eleven feed is a new kind of log-periodic dipole antenna that makes use of a ground plane to form a broad-side far field function [2]. The phase center is fixed in the ground plane and does not move with frequency, unlike to other log-periodic antennas. The name is due to the two-parallel dipoles for each polarization, i.e. an eleven configuration, and many things can be associated with the number eleven for this antenna, like: return loss, more than decade bandwidth, and actually it was initially in 2003 eleven times smaller than its competitor. Nowadays there exist more large-bandwidth alternatives, but they are still not as compact [3]. The eleven antenna could be easily integrated with its balun circuits [4], and cryogenic designs of these antenna are reported [1]. Also, it can give dual polarization performance easily [5].

The basic eleven antenna configuration is two parallel halfwave dipoles above a ground. When the two dipoles are located half wavelength apart, the $E-$ and $H$-plane patterns become equal and thereby there is low cross-polarization in

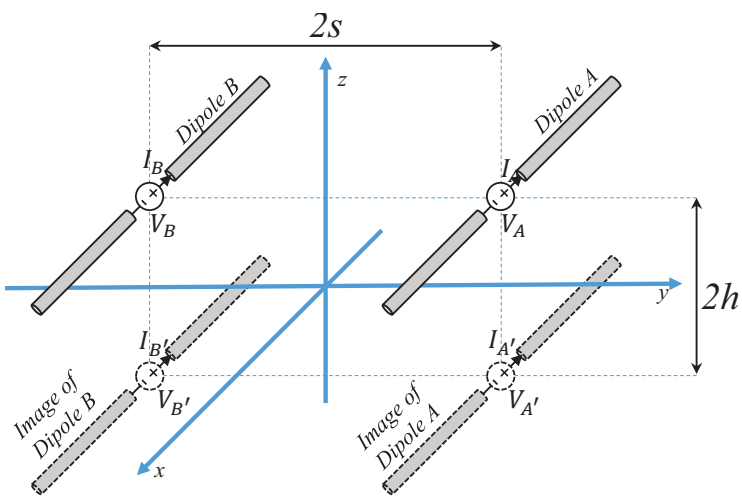

Fig. 1: The eleven Configuration for dipoles $A$, and $B$. The ground plane is removed and the image dipoles are shown. The excitations for image dipoles are dependant on the type of the ground plane (PEC or PMC).

the $45 \mathrm{deg}$ plane. The $12 \mathrm{~dB}$ beam width is about $60^{\circ}$, and the directivity $11 \mathrm{dBi}$, which makes it well suited to feed prime-focus paraboloids. The phase center of this basic simple configuration is located in the ground plane [2].

The present numerical models of the eleven antennas are making use of Perfect Electric Conductor (PEC) ground planes, and to provide the different heights to ground for different dipoles over the frequency band, the dipole array should be tilted over the ground plane. This makes the profile of the antenna high, and seeking for lower-frequencies, the profile becomes even higher.

To have low-profile versions of the eleven antenna it is necessary to, first design the planar array, and second, put the array on a ground plane with reflection phase equal to $0^{\circ}$. Perfect Magnetic Conductor (PMC), which does not exist in nature, provides this characteristic. Hard and soft surfaces, which correspond to parallel strips of PEC, and Artificial Magnetic Conductor (AMC) surface [6], [7], can realize this reflection phase. The integration of the Electromagnetic BandGap (EBG) structures and patch antennas have been studied to enhanced the performance due to the band-gap of surfacewave suppression [8], [9]. EBG structures also been used as ground planes of spiral and curl antennas to achieve low profile designs [10], [11]. The mushroom-like structure, behaves as $\mathrm{EBG}$, and is known to have an effective band-gap for surface- 
TABLE I: Review on the eleven antenna characteristics over PEC and PMC grounds.

\begin{tabular}{|lll|}
\hline Dimensions & over PEC & $h=0.25 \lambda$, and $s=0.25 \lambda$ \\
& over PMC & $h=0.25 \lambda$, and $s \rightarrow 0$ \\
\hline Currents & over PEC & $I_{A}=I_{B}=-I_{A^{\prime}}=-I_{B^{\prime}}=I_{0}$ \\
& over PMC & $I_{A}=I_{B}=I_{A^{\prime}}=I_{B^{\prime}}=I_{0}$ \\
\hline Far-field & over PEC & $4 j \cos \left(\frac{\pi}{2} \sin \theta \sin \phi\right) \sin \left(\frac{\pi}{2} \cos \theta\right) \mathbf{G}_{d x}(\theta, \phi)$ \\
function & over PMC & $4 \cos \left(\frac{\pi}{2} \sin \theta \sin \phi\right) \mathbf{G}_{d x}(\theta, \phi)$ \\
\hline E-plane & over PEC & $4 j \cos \left(\frac{\pi}{2} \sin \theta \sin \phi\right) \sin \left(\frac{\pi}{2} \cos \theta\right) \mathbf{G}_{d x}(\theta, 0)$ \\
pattern & over PMC & $4 \mathbf{G}_{d x}(\theta, 0)$ \\
\hline H-plane & over PEC & $4 j \cos \left(\frac{\pi}{2} \sin \theta \sin \phi\right) \sin \left(\frac{\pi}{2} \cos \theta\right) \mathbf{G}_{d x}\left(\theta, \frac{\pi}{2}\right)$ \\
pattern & over PMC & $4 \cos \left(\frac{\pi}{2} \sin \theta\right) \mathbf{G}_{d x}\left(\theta, \frac{\pi}{2}\right)$ \\
\hline
\end{tabular}

wave propagation, which can be useful to improve antenna radiation patterns [12].

In this paper, the theory of the eleven antenna on PMC ground is investigated in Section II. Some results for far-field function of the antenna are extracted. In Section III, the theory is used to analyze the characteristics of a pair of printed folded dipoles. At last, a planar array of folded-dipoles is designed, and its performance is studied, and a brief discussion of the realization of the AMC ground is given. At last, Section IV gives some concluding remarks.

\section{THEORY}

Here, the basic theory for the eleven configuration over PEC and $\mathrm{PMC}$ ground planes is reviewed. The eleven configuration for two dipoles is shown in Fig. 1. Two real dipoles $A$, and $B$, with a separation of $2 s$, are located in the height $h$ over a PEC (or PMC) ground. Based on image theory, one can remove the ground and put two image dipoles $A^{\prime}$, and $B^{\prime}$ in the height $h$ under the ground plane.

For the eleven structure over PEC ground the image currents are as

$$
I_{A}=I_{B}=-I_{A^{\prime}}=-I_{B^{\prime}}=I_{0},
$$

and in the case of PMC ground,

$$
I_{A}=I_{B}=I_{A^{\prime}}=I_{B^{\prime}}=I_{0} .
$$

It is necessary to mention that for a $x$-directed isolated dipole, the far-field function is [13]

$$
\mathbf{G}_{d x}(\theta, \phi)=\frac{-j}{2 \pi} \eta I_{0}(\cos \theta \cos \phi \hat{\boldsymbol{\theta}}-\sin \phi \hat{\boldsymbol{\phi}}) \tilde{j}(\theta, \phi),
$$

where

$$
\tilde{j}(\theta, \phi)=\frac{\cos \left(\frac{\pi}{2} \sin \theta \cos \phi\right)}{1-(\sin \theta \cos \phi)^{2}}
$$

in the next two subsections the far-field function for each case is calculated.

\section{A. The eleven Antenna over PEC Ground}

The array dimensions would be considered as

$$
h=0.25 \lambda, s=0.25 \lambda .
$$

For the upper half space $\left(z>0\right.$ or $\left.|\theta|<\frac{\pi}{2}\right)$, the far-field function for this case is

$$
\mathbf{G}^{\mathrm{PEC}}=4 j \cos \left(2 \pi \frac{s}{\lambda} \sin \theta \sin \phi\right) \sin \left(2 \pi \frac{h}{\lambda} \cos \theta\right) \mathbf{G}_{d x}(\theta, \phi),
$$

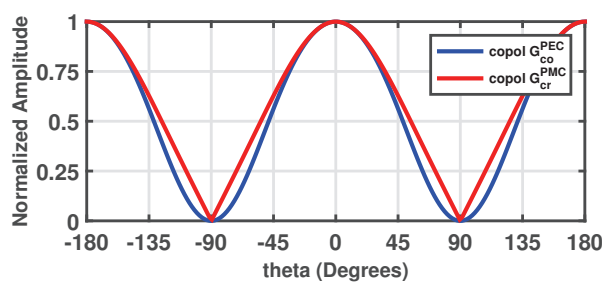

(a)

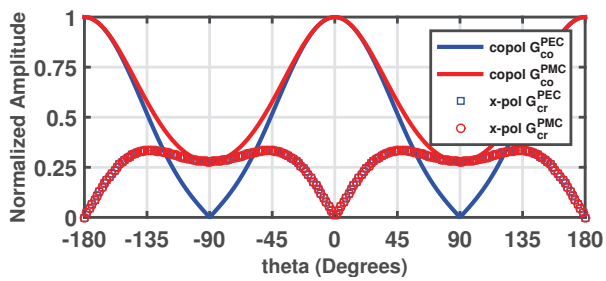

(b)

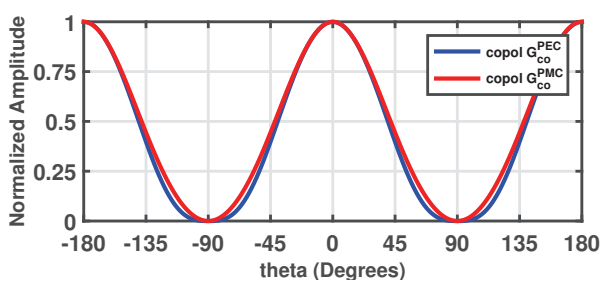

(c)

Fig. 2: Normalized amplitude of far-field function for the eleven antenna over PEC, and PMC ground planes. (a) copolarization at $\phi=0^{\circ}(E-)$ plane, (b) co-, and crosspolarization at $\phi=45^{\circ}(D-)$ plane, and (c) co-polarization at $\phi=90^{\circ}(H-)$ plane.

which with the dimensions as (5), it is rewritten as

$$
\mathbf{G}^{\mathrm{PEC}}=4 j \cos \left(\frac{\pi}{2} \sin \theta \sin \phi\right) \sin \left(\frac{\pi}{2} \cos \theta\right) \mathbf{G}_{d x}(\theta, \phi) .
$$

\section{B. The eleven Antenna over PMC Ground}

in the case of the PMC ground plane we would set

$$
s=0.25 \lambda, h \rightarrow 0 .
$$

For the far-field function like the latter case

$$
\mathbf{G}^{\mathrm{PMC}}=4 \cos \left(2 \pi \frac{s}{\lambda} \sin \theta \sin \phi\right) \cos \left(2 \pi \frac{h}{\lambda} \cos \theta\right) \mathbf{G}_{d x}(\theta, \phi),
$$

which with dimensions of (7),

$$
\mathbf{G}^{\mathrm{PMC}}=4 \cos \left(\frac{\pi}{2} \sin \theta \sin \phi\right) \mathbf{G}_{d x}(\theta, \phi)
$$

\section{Comparison of two cases}

A quick review of two studied cases is presented in TABLE I. Here, some simulation and calculation results are studied for far-field functions.

Normalized amplitude of co-polar component of $\mathbf{G}^{\mathrm{PEC}}$ in (6), and $\mathbf{G}^{\mathrm{PMC}}$ in (8), is plotted in Fig. 2. Three main planes $E-, D-$, and $H-$ planes $\left(\phi=0, \frac{\pi}{4}\right.$, and $\left.\frac{\pi}{2}\right)$ are plotted. In the $\phi=\frac{\pi}{4}$ plane, the cross-polar component is shown together with the co-polar component. 


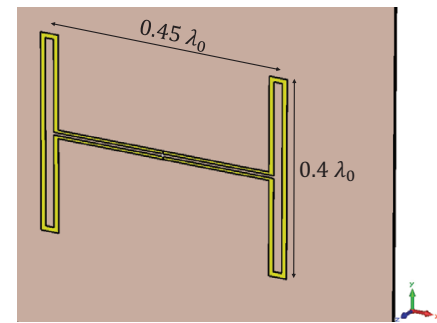

Fig. 3: The basic folded Dipoles in the eleven configuration. Two basic parameters are shown.

TABLE II: CST simulation results for input impedance and directivity of the antenna in Fig. 3

\begin{tabular}{|c|c|c|}
\hline & Input impedance & Directivity \\
\hline without ground plane & $67+j 62 \Omega$ & $6 \mathrm{dBi}$ \\
\hline PEC ground plane & $46+j 14 \Omega$ & $11.2 \mathrm{dBi}$ \\
\hline PMC ground plane & $42+j 64 \Omega$ & $9.2 \mathrm{dBi}$ \\
\hline
\end{tabular}

It is obvious from (6) that for $\left|\mathbf{G}^{\mathrm{PEC}}\right|$ there is no radiation in the direction of $|\theta|=\frac{\pi}{2}$ for all $\phi$ s. However, $\left|\mathbf{G}^{\mathrm{PMC}}\right|$, is zero for just $\phi=0, \frac{\pi}{2}$, and not in other planes. This means that there is some radiation in the array plane, and it decreases the directivity of the antenna in this case. In the other words, putting an eleven antenna on a PMC ground yields lower directivity in comparison with the case of the PEC ground. This fact would be shown in the next section.

\section{RESUlts FOR FOLDED PRINTED DiPOLES}

Folded dipoles are often used in the eleven antenna in order to obtain wide-band performance by cascading one after another in a log-periodic array. The impedance matrix of the folded dipoles under the eleven configuration using a two-mode method with including the mutual couplings is investigated in [14].

\section{A. Single Pair of Folded Dipoles in the eleven Configuration}

A single pair of folded dipoles are shown in figure 3. A RO4003 board $\left(\epsilon_{r}=3.55, \tan \delta=2.7 \times 10^{-3}\right)$ with thickness of $1.524 \mathrm{~mm}$ is used, and the antenna is simulated in CST software. Three cases are studied: (a) without ground plane, (b) over PEC ground plane, (c) over PMC ground plane. The height to the ground in the PEC case is quarter-wavelength and in PMC case is zero. A magnetic boundary condition $\left(H_{t}=0\right)$ is used to model infinite magnetic ground.

The co-polar directive gain patterns for these three cases are plotted in Fig. 4. Because of the symmetry, only half of the patterns $\left(\theta>0^{\circ}\right)$ are shown. In the PEC and PMC cases, there is no radiation for $\theta>90^{\circ}$. Three main planes $\phi=$ $0^{\circ}, 45^{\circ}, 90^{\circ}$ are considered, and the cross-polar directive gain in $\phi=45^{\circ}$ is also plotted.

One may notice that for the PMC case, the directive gain in the $\theta=90^{\circ}$ direction is not zero (in-plane radiation), and even larger than in the free-space case. This is due to nonzero height of the substrate and surface-wave propagation (TM mode) in the substrate. This radiation decreases the maximum directive gain in this case. TABLE II shows that the PEC case

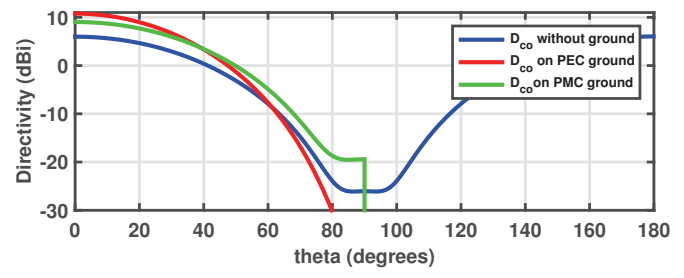

(a)

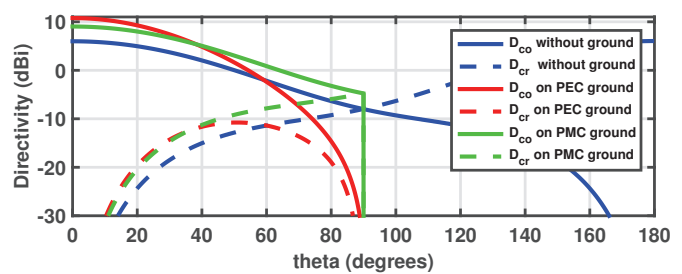

(b)

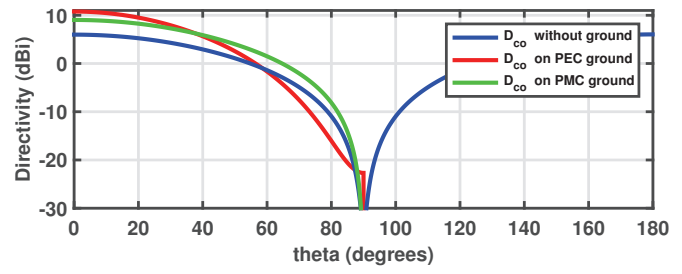

(c)

Fig. 4: Directive gain pattern for single pair of printed folded dipoles in the eleven configuration shown in Fig. 3. (a) copolarization at $\phi=0^{\circ}(E-)$ plane, (b) co-, and crosspolarization at $\phi=45^{\circ}(D-)$ plane, and (c) co-polarization at $\phi=90^{\circ}(H-)$ plane.

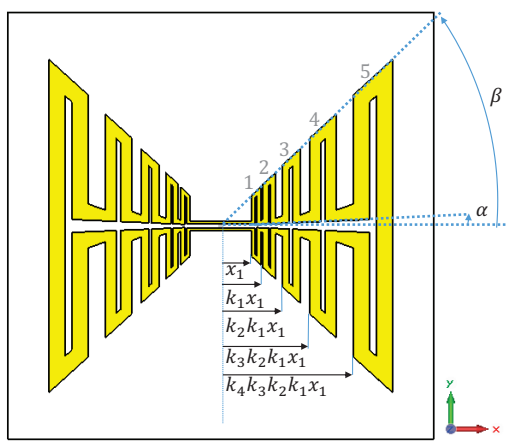

Fig. 5: Planar array of five folded dipoles in the eleven configuration. Geometry parameters are shown.

has $11 \mathrm{dBi}$ directivity, but in the PMC case, because of the in-plane radiation, only $9 \mathrm{dBi}$ is achieved.

TABLE II also shows the input impedance values for connected two folded dipoles in Fig. 3.

\section{B. Five Pairs of Folded Dipoles in the eleven Configuration}

Five pairs of folded dipoles are connected in eleven configuration on RO4003 substrate as Fig. 5. Four scaling factors $\left(k_{1}-k_{4}\right)$, and two angle parameters $(\alpha, \beta)$ are used to define new dipoles. Design parameters and their values are listed in TABLE III. 
TABLE III: Design parameters for the array shown in Fig. 5

\begin{tabular}{|cc|cc|}
\hline Parameter & Value & Parameter & Value \\
\hline$k_{1}$ & 1.3795 & $x_{1}$ & $19.92 \mathrm{~mm}$ \\
\hline$k_{2}$ & 1.4485 & $\beta$ & $42.18^{\circ}$ \\
\hline$k_{3}$ & 1.4485 & $\alpha$ & $2.5^{\circ}$ \\
\hline$k_{4}$ & 1.4888 & & \\
\hline
\end{tabular}

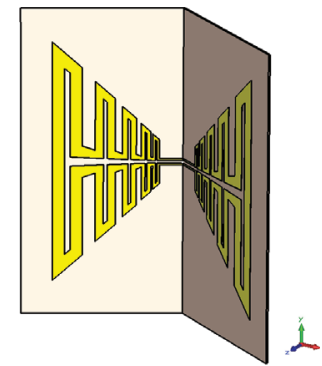

Fig. 6: Tilted Array of folded dipoles over PEC ground.

Six cases are studied here:

1) Planar array without any ground,

2) Planar array on PMC ground with zero-height,

3) Tilted array on PEC ground: the array is tilted to provide quarter-wavelength height to the ground. The tilted array is shown in Fig. 6. The tilted structure has large profile, and although our main goal is to design planar eleven array, the results from the tilted array over PEC ground are a good reference to evaluate the effectiveness of our approach.

4) Planar array on infinite PEC ground with quarterwavelength-height: the planar antenna is on an infinite PEC ground plane. the height is quarter-wavelength $\left(\frac{\lambda_{0}}{4}\right)$ at center frequency $f_{0}=1.1 \mathrm{GHz}$.

5) Planar array on finite PEC ground with quarterwavelength-height: like the previous case, but the ground is finite, with the size equal to array.

6) Planar array on bed-of-the-nails: the bed-of-the-nails is shown in the Fig. 7. rectangular pins with the size $0.009 \lambda_{0} \times 0.009 \lambda_{0} \times 0.25 \lambda_{0}$ are used as nail, with a pin-to-pin separation equal to $0.02 \lambda_{0}$. These parameters are used from manufacturing limitations.

The design frequency band is $0.6-1.5 \mathrm{GHz}$. In each case, the antenna could be matched to $100 \Omega$ with a good enough $\left|S_{11}\right|$ level. The return loss plots are shown in Fig. 8. However, it is possible to perform some optimizations to get better

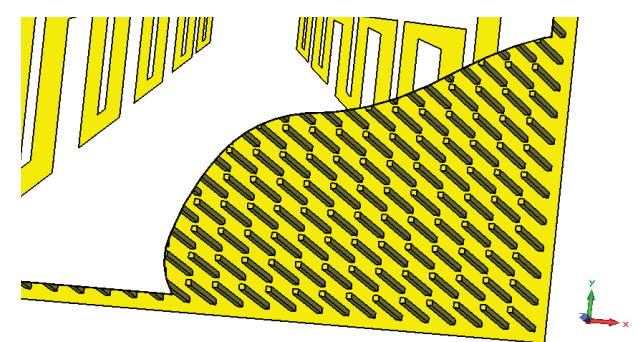

Fig. 7: Planar Array over bed-of-the-nails ground.

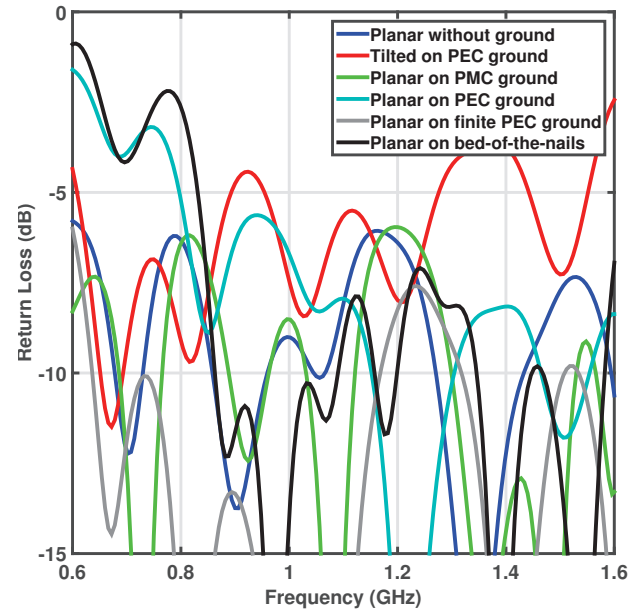

Fig. 8: Return loss for array in different cases.

matching.

Both return-loss and directivity should be taken in to account to quantify the operating bandwidth. From this point of view, the bandwidth of the planar eleven over infinite PMC ground is $0.7-1.45 \mathrm{GHz}(\approx 2: 1$, or $70 \%)$.

The directivity versus frequency for the array is also shown in Fig. 9. Like the single-pair case in TABLE II, in the presence of PMC ground, the directivity is about $9 \mathrm{dBi}$ in the frequency band. Also From TABLE II, and Fig. 9, one can say that in the absence of the ground plane, the directivity for the planar array over the frequency band is close to the single-pair antenna (about $6 \mathrm{dBi}$ ). However, the tilted array on PEC ground has a directivity a little less than $11 \mathrm{dBi}$. Authors believe that performing some extra optimizations can improve the directivity to $11 \mathrm{dBi}$ for tilted array on PEC ground, too.

The directivity for the fourth case (flat array on infinite PEC ground with quarter-wavelength-height) is close to tilted array's directivity, however, the return losses are not similar. Furthermore, the quarter-wavelength solution works around the center frequency, and is not suitable for higher or lower frequency bands. However, compared to third case, because of not having surface waves, the directivity for the fourth case is higher. This shows the importance of blocking the surface waves to have better directivity. On the other hand, if one uses a finite PEC ground plane (fifth case) instead of infinite one, there would be $1-2 d B i$ loss in directivity. However, the directivity is even higher than the infinite PMC case, again it is due to strong surface waves on the PMC ground.

The black curve in the Fig. 9 shows the directivity for the planar array on the bed of the nails ground plane. It shows that in a narrow bandwidth, it works like the ideal PMC ground, and the curve drops dramatically in lower frequencies. One can say that although the bed-of-nails can realize the PMC behavior in a narrow bandwidth, it cannot block the surface waves.

Here, a five element array is presented, however, it seems that it is possible to increase the number of the elements 


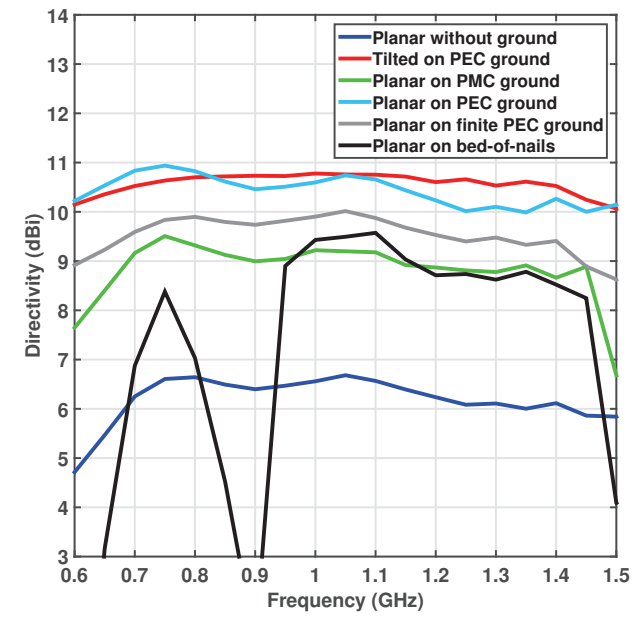

Fig. 9: Directivity for array in different cases.

to have wider bandwidths. The possibility to increase the bandwidth by adding more elements, makes the eleven configuration an interesting idea. However, different coupling mechanisms between one elements and the other elements and/or the ground plane, force the antenna designer to be more careful about the design procedure. Unfortunately, it is mostly hard to model these couplings, and in practice one should perform some optimizations to find the best performance for the array. For example, in the presented array, different scaling factors are optimized to have the best and most stable matching and directivity over the frequency band.

\section{Discussion on the Realization of $A M C$}

A PMC surface can provide zero-degree reflection phase. As in previously reported eleven arrays [1]-[4], a simple way to achieve this reflection phase is putting the antenna over a PEC ground with a quarter-wavelength height. However, this solution only works for a single frequency and to increase the bandwidth, the antenna should be tilted. Furthermore, the profile of antenna would be larger if someone seeks for lower frequency bands. As proposed in this paper, low-profile planar eleven array design is promising if we can realize the AMC performance. The realization of the AMC ground plane for low-profile antenna designs has been the subject of many works [10]-[12], in which they have tried to get the zerodegree reflection phase.

Results in the previous subsections showed that the surface wave in the substrate over the PMC ground, degrades the directivity of the antenna. The surface wave propagates in TM mode, so to overcome this problem one can use mushroomlike AMCs which can block TM surface waves. This will be done in future works, to combine planar eleven array with the mushroom-like AMC [12]. The EBG structures usually do not have band-gap performance over a wide-band. An idea is to scale the EBG cells, so each array element faces EBG cells which are designed in the same frequency as the resonant frequency of that element.

\section{CONCLUSION}

The idea of the eleven antenna has many potentials to achieve ultra-wide-band performances. This paper presents a detailed study of the theory of the eleven configuration over PMC ground plane. In previous eleven antennas, the designer was forced to tilt the array over a PEC ground, and the profile of the antenna was high. It is revealed that to lower the profile of the antenna, PMC ground could be used. The simulation results for the planar array of folded dipoles in the eleven configuration on an ideal PMC ground shows that this idea is promising, and low-profile array is possible, if the PMC can be realized for this application.

However, the propagation of the surface waves in the substrate in the presence of PMC ground plane, degrades the antenna radiation performance. Authors believe that using EBG structures as ground plane can provide the AMC performance together with the surface-wave blockage. As the EBG structures usually may not have a wide-band performance, one may need to scale the EBG cells as well as the dipoles.

\section{REFERENCES}

[1] J. Yang, M. Pantaleev, P.-S. Kildal, B. Klein, Y. Karandikar, L. Helldner, N. Wadefalk, and C. Beaudoin, "Cryogenic 2-13 ghz eleven feed for reflector antennas in future wideband radio telescopes," Antennas and Propagation, IEEE Transactions on, vol. 59, no. 6, pp. 1918-1934, 2011.

[2] R. Olsson, P.-S. Kildal, and S. Weinreb, "The eleven antenna: a compact low-profile decade bandwidth dual polarized feed for reflector antennas," Antennas and Propagation, IEEE Transactions on, vol. 54, no. 2, pp. 368-375, 2006.

[3] P.-S. Kildal, "Some reflector and feed antenna inventions that made a difference: Fundamentals and examples from radio telescopes, satellite communications and radio links," in AFRICON, 2013. IEEE, 2013, pp. $1-3$.

[4] H. Raza, J. Yang, and M. Pantaleev, "Integration of ultra-wideband planar baluns into the eleven feed," Microwaves, Antennas \& Propagation, IET, vol. 8, no. 1, pp. 22-28, 2014.

[5] J. Yang, X. Chen, N. Wadefalk, and P.-S. Kildal, "Design and realization of a linearly polarized eleven feed for 1-10 ghz," Antennas and Wireless Propagation Letters, IEEE, vol. 8, pp. 64-68, 2009.

[6] P.-S. Kildal, "Definition of artificially soft and hard surfaces for electromagnetic waves," Electronics Letters, vol. 24, no. 3, pp. 168-170, 1988.

[7] _ , "Artificially soft and hard surfaces in electromagnetics," Antennas and Propagation, IEEE Transactions on, vol. 38, no. 10, pp. 1537-1544, 1990.

[8] R. Coccioli, F.-R. Yang, K.-P. Ma, and T. Itoh, "Aperture-coupled patch antenna on uc-pbg substrate," Microwave Theory and Techniques, IEEE Transactions on, vol. 47, no. 11, pp. 2123-2130, 1999.

[9] F. Yang and Y. Rahmat-Samii, "Microstrip antennas integrated with electromagnetic band-gap (ebg) structures: A low mutual coupling design for array applications," Antennas and Propagation, IEEE Transactions on, vol. 51, no. 10, pp. 2936-2946, 2003.

[10] T. Liu, W. Zhang, M. Zhang, and K. Tsang, "Low profile spiral antenna with pbg substrate," Electronics Letters, vol. 36, no. 9, pp. 779-780, 2000.

[11] F. Yang and Y. Rahmat-Samii, "A low-profile circularly polarized curl antenna over an electromagnetic bandgap (ebg) surface," Microwave and Optical technology letters, vol. 31, no. 4, pp. 264-267, 2001.

[12] - "Reflection phase characterizations of the ebg ground plane for low profile wire antenna applications," Antennas and Propagation, IEEE Transactions on, vol. 51, no. 10, pp. 2691-2703, 2003.

[13] P. Kildal, "Foundations of antenna engineering: A unified approach for line-of-sight and multipath," Kildal Antenn AB, 2015.

[14] J. Yang, D. Nyberg, and J. Yin, "Impedance matrix of a folded dipole pair under eleven configuration," IET Microwaves, Antennas \& Propagation, vol. 4, no. 6, pp. 697-703, 2010. 